\title{
Mountain biking injuries in rural England
}

\author{
L M Jeys, G Cribb, A D Toms, S M Hay
}

\begin{abstract}
Background-Off road mountain biking is now an extremely popular recreation and a potent cause of serious injury.

Aim-To establish the morbidity associated with this sport.

Methods-Data were collected prospectively over one year on all patients presenting with an injury caused by either recreational or competitive off road mountain biking.

Results-Eighty four patients were identified, 70 males and 14 females, with a mean age of 22.5 years (range 8-71). Most accidents occurred during the summer months, most commonly in August. Each patient had an average of 1.6 injuries $(n=$ 133) and these were divided into 15 categories, ranging from minor soft tissue to potentially life threatening. Operative intervention was indicated for 19 patients $(23 \%)$ and several required multiple procedures. The commonest injuries were clavicle fractures $(13 \%)$, shoulder injuries $(12 \%)$, and distal radial fractures $(11 \%)$. However, of a more sinister nature, one patient had a $\mathrm{C} 2 / 3$ dislocation requiring urgent stabilisation, one required a chest drain for a haemopneumothorax, and another required an emergency and life saving nephrectomy.

Conclusion-This sport has recently experienced an explosion in popularity, and, as it carries a significant risk of potentially life threatening injury across all levels of participation, the use of protective equipment to reduce this significant morbidity may be advisable.

(Br F Sports Med 2001;35:197-199)
\end{abstract}

Trauma Unit,

Orthopaedic Surgery

Department, Royal

Shrewsbury Hospital, Mytton Oak Road,

Shrewsbury, UK

L M Jeys

G Cribb

A D Toms

S M Hay

Correspondence to:

Dr Jeys, 27 Newton Park

Road, West Kirby, Wirral

CH48 9XE, UK

lee.jeys@btclick.com

Accepted 23 February 2001
Keywords: mountain biking; injury

Within the past 10 years, off road mountain cycling has become an extremely popular recreation throughout the western world. In addition to recreational appeal, it has evolved into a popular and highly competitive sport, achieving Olympic status for Sydney 2000.

Recent articles have shown that injuries sustained while cycling caused 35000 emergency admissions in 1991-1995. ${ }^{1}$ The physical geography of the United Kingdom is ideally suited to the sport, and there has been a gradual but significant increase in mountain biking injuries, some of which are very serious and even life threatening. We have therefore evaluated both the types and severity of injuries seen in a rural district general hospital.

\section{Method}

Data were collected prospectively over one year. All injuries caused by off road mountain cycling referred to the orthopaedic trauma unit at the Royal Shrewsbury Hospital were included. Patients were either seen acutely in the accident and emergency department or subsequently in the fracture clinic.

Injuries were identified in 84 patients, who had been using their bikes either recreationally or competitively. The information retrieved included basic details (name, age, and sex), the types of injuries sustained, the need for admission/surgery, and any associated complications. The injury types were then classified into 16 categories according to the severity and region of injury, to facilitate data handling.

\section{Results}

During the period studied, 84 patients with mountain biking injuries were referred to the orthopaedic trauma unit. The vast majority of injuries occurred in patients engaged in the sport for recreational rather than competitive purposes and these were predominantly male $(\mathrm{n}=70,83 \%)$. The patient age range was $8-71$ years, but the main cohort were from the 8-35 year age group, with a mean age of 22.5 years (fig 1). A total of 133 injuries were recorded, ranging from one to six injuries per patient (mean $=1.6$ injuries/patient). Most were sustained in the late summer months, usually August (fig 2).

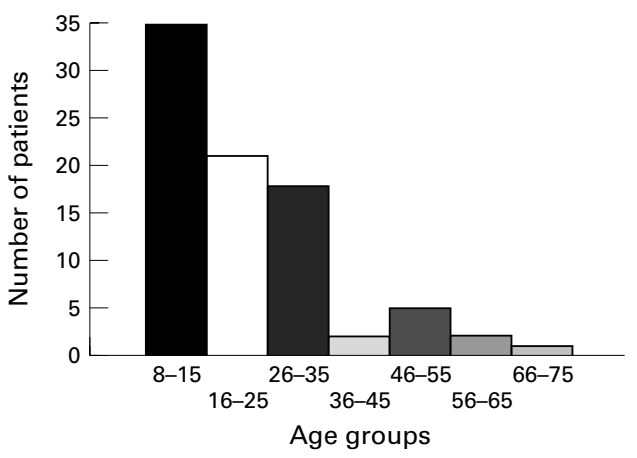

Figure 1 Distribution of age of patients. 


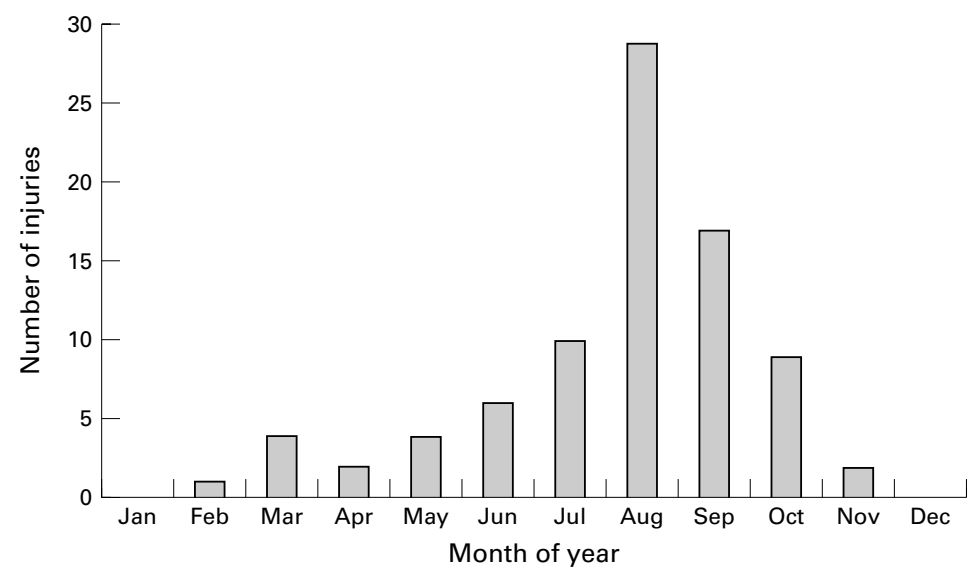

Figure 2 Distribution of injuries throughout the year.

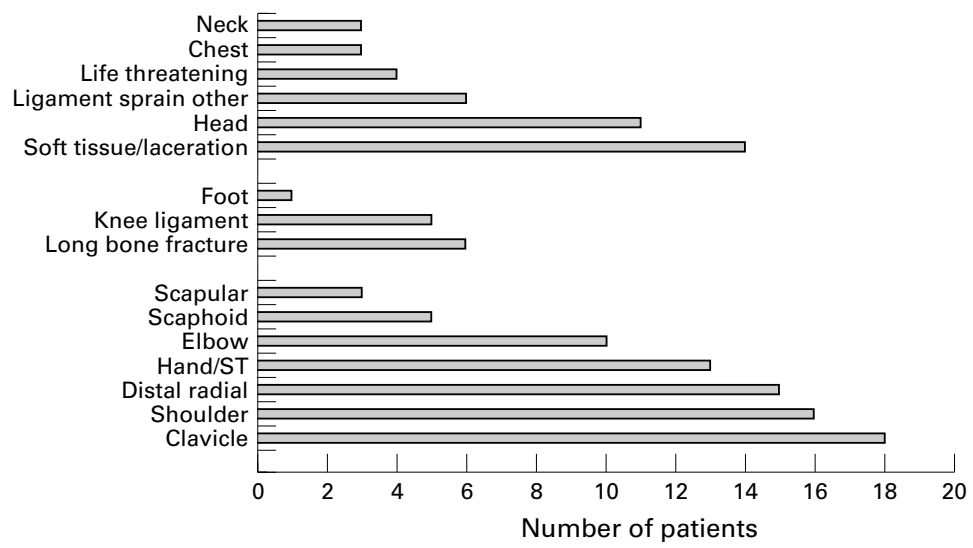

Figure 3 Classification of injuries.

A total of 19 patients needed operative treatment $(23 \%)$, some requiring multiple procedures with a prolonged hospital stay. The injuries ranged from soft tissue lacerations to life threatening injuries, and were distributed as shown in fig 3. The most common injury was fracture of the clavicle (13\%), although this was closely followed by other shoulder girdle injuries $(12 \%)$, distal radial fractures $(11 \%)$, and soft tissue injury/laceration $(10 \%)$.

This represents a spectrum of injuries commonly handled in fracture clinics, but in addition some more serious, even life threatening, injuries were identified. These included six patients with open and closed fractures of the femur or tibia, one of whom, an 11 year old, also had a significant head injury requiring helicopter transfer to the regional neurosurgical centre. In addition, one patient sustained neurological deficit, with a C2/3 fracture dislocation, and required urgent stabilisation. A further patient required a life saving nephrectomy to control haemorrhaging, another patient required drainage of a life threatening haemopneumothorax, and a further sustained a serious open fracture dislocation of the dominant carpus, which often results in significant long term morbidity.

\section{Discussion}

Recreational cycling was the third most popular activity in the United States in 1998, with 8.6 million people riding their bikes off road in
1998 compared with 4.59 million in $1993 .^{2}$ This represents a staggering increase of $87 \%$ in five years. The British Cycling Federation estimates that 10000 mountain bikers compete on a regular basis. The sale of mountain bikes has increased dramatically over the last 20 years, and in 1994 accounted for $66 \%$ of the adult retail market in the United Kingdom. ${ }^{3}$

Off road cycling often involves travelling at speed down steep often unpredictable inclines and through narrow tracks covered in loose stone. It is the excitement of this unpredictable and dangerous challenge that motivates the predominantly young male participants, and it is not surprising therefore that both the experienced and the inexperienced rider will frequently fall. Such accidents may be high energy in nature and may result in serious injury, as this study illustrates. Indeed, several case reports of individual serious injuries caused by the use of mountain bikes have been published. $^{4-6}$ However, this is the first data series representing a collective spectrum of injuries occurring in the United Kingdom, a country where the popularity of the sport and the incidence of related injuries has grown enormously in recent years.

Previous reports from the United States and New Zealand have indicated the extensive use of helmets by cyclists $(80-88 \%$ in off road riders), ${ }^{78}$ possibly accounting for a low incidence of head and neck injuries. They concluded that most off road injuries were minor, ${ }^{78}$ and that the incidence of fractures was low. ${ }^{7}$ However, our study found that head and neck injuries accounted for $10 \%$ of injuries. Moreover fractures, including those of both the upper and lower limbs, occurred in $45 \%(n=38)$ of patients referred.

Injury to the upper limb is a predictable result of a fall from a bicycle, and this is reflected in this series by a high incidence of fractures of the clavicle, shoulder injuries, and distal radial fractures. In a fall, the apex of the shoulder is often the site of initial impact, consistent with acromioclavicular joint disruption, clavicular fracture, and shoulder dislocation. Predictably the elbow is not spared and fractures about this joint were also recorded.

The less common but much more serious injuries include internal organ rupture, head injury, neck injury, and long bone fracture. These represent $20 \%$ of injuries referred during the study period. To date no deaths have been recorded in our series, but, given the nature of the forces involved and the energy imparted on impact, future mortality in this continuing analysis is expected.

Although at face value the injuries may not individually seem particularly serious, they each create personal morbidity, including distress, and medical/surgical work, and also have a socioeconomic cost; these are serious problems resulting from what is essentially a leisure pursuit.

The seasonal variation in the distribution of injuries was striking, but perhaps not surprising. Most injuries occurred during the summer months, when conditions for off road cycling are most suitable. 


\section{Conclusion}

Within the growing culture of leisure and exercise, off road cycling or mountain biking has become a convenient, pleasurable, and extremely popular pastime. This study has identified and categorised the spectrum of associated injuries, some of which are serious and may cause significant morbidity. We believe that these injuries have resource implications for rural hospitals that did not exist before the recent increase in this sport.

Given the findings of the study, it is important that doctors confronted with a mountain biking injury take the mechanism of injury into account and prepare for significant trauma. Injuries are usually sustained by high velocity impact into immovable objects with little or no patient protection. Further investigations are being carried out into the prevalence and effectiveness of the use of body armour by both recreational and competitive mountain bikers. This may prove a valuable step in improving the safety of this sport.
We thank Mr H Nassar for data retrieval, Mrs Ellen Stockton for secretarial support and data handling, and the reception staff of the accident and emergency department of the Royal Shrewsbury Hospital for data retrieval.

Contributors: L J, substantial contribution to design, acquisition of data, and drafting of article; G C, substantial contribution to acquisition of data and revising article; A T, substantial contribution to concept and acquisition of data; $\mathrm{S} \mathrm{H}$, substantial contribution to concept, drafting, revision, and final substantial contrib
approval of paper.

1 Cook A, Sheikh A. Trends in serious head injuries among cyclists in the UK. BMF 2000;321:1055-6.

2 Sports participation in 1998. New York: National Sporting Goods Association, NPD Research, 2000.

3 Bicycle Keynote Report. Maidstone: The Chartered Institute of Marketing, 1995.

4 Cabezas-Checci C. High flow priapism after perineal trauma. Actas Urol Esp 1998;22:359-61.

5 Nehoda H, Hochleitner BW. Subcapsular liver haematomas caused by bar end in mountain bike crashes. Lancet 1998; 351:342.

6 Lovell ME. Mountain bike injury to the abdomen, transection of the pancreas and small bowel evisceration. Injury 1992;23;499-500.

7 Rivara FP. Injuries involving off road cycling. 7 Fam Pract 1997;44:481-5.

8 Pfeiffer RP. Off-road cycling injuries. An overview. Sports Med 1995;19;311-25.

\section{Take home message}

Mountain biking is a popular, exciting, and challenging sport, enjoyed by all ages and abilities. Injuries occur and these may be potentially serious, even life threatening. The prevalence and effectiveness of the use of body armour in the sport requires investigation.

\section{Commentary}

The paper highlights the hazards of an increasingly popular form of cycling. I was surprised to discover that, of the 84 patients attending the accident and emergency department, four (5\%) had potentially life threatening injuries. From these figures, it may easily be concluded that mountain biking is an extremely hazardous pursuit; however, in my experience the vast majority of falls from all types of cycle fortunately result in nothing more than minor abrasions and do not require attendance at an accident and emergency department.

It is propitious that leisure mountain biking has a culture where the wearing of purpose built protective helmets is the rule rather than the exception-fashion and safety unusually walking hand in hand. Helmets have always been compulsory for all competitive events. The authors suggest the possible use of some form of body armour as a means of reducing injury, and this is currently used only in the downhill racing discipline of mountain biking. In this, competitors carry out an individual time trial from the top to the bottom of a steep hill or mountain via a circuitous, poorly surfaced, and highly "technical" course. In the more popular cross country discipline, considerations of weight, reduced mobility, and decreased heat loss would make contemporary protective clothing impractical.

From the ages of those presenting to the department, it will be apparent that mountain biking is an inclusive sport accessible to a broad range of ages and abilities. The level of skill necessary for some courses is high, and youth may just over reach itself. When the risks of the off road sport are being assessed, it should be noted that many participants are using off road trails in the belief that they are safer. They would contend that tarmac roads have ceased to be a cyclist friendly environment, rather a place where they are obliged to inhale exhaust fumes and put themselves at the mercy of other road users. 